\title{
USER-EQUILIBRIUM ROUTE SET ANALYSIS OF A LARGE ROAD NETWORK*
}

Hillel Bar-Gera, Ben-Gurion University of the Negev, Be'er Sheva, Israel, and

David Boyce, Northwestern University, Evanston, Illinois, USA

\section{INTRODUCTION}

By 1953 Martin Beckmann had formulated and analyzed the static, deterministic userequilibrium (UE) route choice model with variable origin-destination flows (demand) (Beckmann et al, 1956; Boyce, 2004). Twenty years later, Suzanne Evans (1973, 1976) proposed and analyzed an algorithm for solving a version of Beckmann's model that corresponded to practitioner models with origin-destination (OD) constraints and transit as well as road modes. The effectiveness of her algorithm for large networks was studied by several researchers; see Boyce and Bar-Gera (2004) for a recent review. Other young researchers at that same time proposed and tested algorithms for solving the user-equilibrium problem with fixed OD flows (LeBlanc et al, 1975; Nguyen, 1974; Florian and Nguyen, 1976), building upon the pioneering theoretical research of Jorgensen (1963), Dafermos (1968) and Brynooghe et al (1969). More recently, Patriksson (1994) thoroughly synthesized the accumulated literature from this 40-year research effort, and proposed his own userequilibrium algorithms for fixed and variable flow problems (Larsson and Patriksson, 1992; Lundgren and Patriksson, 1998). Nagurney (1999) synthesized a related literature on the variational inequality approach to a family of problems, including user-equilibrium; Florian and Hearn (1999) offered their review, which includes recent developments on road pricing.

Despite these highly creative and sustained efforts over a 50-year period, as well as steady progress in applying some of these findings to practical problems of urban travel forecasting, effectively nothing is known about the characteristics of the route sets that comprise the solution of the UE model for a large road network. Basic questions, such as the following, remain unanswered:

\footnotetext{
* Reproduced from H. S. Mahmassani (2005) Transportation and Traffic Theory: Flow, Dynamics and Human Interaction, Proceedings of the 16th International Symposium on Transportation and Traffic Theory, Chapter 34, Elsevier, Amsterdam, pp. 673-692.
} 
1. What are the number, range and frequency of routes per OD pair in the UE solution, that is a solution in which all used routes have equal travel costs, and no unused route has a lower travel cost?

2. How does the number of routes per OD pair vary with OD generalized travel cost, OD flow and direction from each origin to destination with respect to the road network?

These questions are the focus of this chapter. Such questions are important, not only because they probe the basic properties of this commonly applied route choice model; they also help us to assess whether the model is a sound choice for forecasting future travel. Moreover, knowing the answers to these questions could stimulate empirical research on corresponding questions concerning actually used routes, which would provide for the first time an effective basis for route choice model validation. See Bovy and Stern (1990) for an extensive review and analysis of route choice behaviour from many viewpoints.

Several additional questions should be addressed eventually, but are not considered here:

1. How different are the routes (sequences of links) that comprise multiple-route sets between OD pairs? Harris (2002) made an initial exploration of such route sets using data similar to those analyzed here.

2. As the route flows in the deterministic UE solution are not unique (Patriksson 1994, p.44), what is the range and distribution of UE route flows among alternative solutions; how do they compare with most likely UE route flows?

3. How does the UE route set vary with the definition and parameter values of both the generalized cost function and the deterrence function in the UE model, which partially determine the level of congestion in the road network?

4. How is the route set affected by the topology, detail and coding conventions of the road network, such as with respect to turns and turn penalties, and the structure and level of service of the transit network?

5. How is the route set affected by the pattern of origin-destination flows?

For all of the questions listed above, we are interested in answers for a highly converged solution. Moreover, the answers should not be affected by limitations of the solution algorithm, such as on the number of routes in the solution, for example. The capability to solve the static, user-equilibrium problem precisely for both small and large networks became available for fixed OD flows only in 1999 with the publication of the Origin-Based 
Assignment (OBA) algorithm by Bar-Gera (1999, 2002). An extension to the case of variable OD flows over multimodal networks was presented by Bar-Gera and Boyce (2003). To our knowledge, only the OBA algorithm is capable of producing solutions with the detail and precision necessary to answer the questions listed above.

Our approach to answering these questions may be described as exploratory and intuitive. Given a single, highly-converged solution of a single-class, two-mode, user-equilibrium model with negative exponential deterrence functions for the Chicago Region, we explore the first two questions listed above. We emphasize that our findings pertain to this one example. Our tools of analysis are spreadsheets and graphs, relying heavily on visual expressions of the results to formulate research hypotheses and reach tentative conclusions. The analyses were initially more detailed, and then aggregated for this synthesis.

The presentation of the results is organized as follows. First, a brief, technical description of the model is offered, together with parameter values and overall summary measures such as mean generalized costs by mode, total regional flow by mode, etc. Certain properties of the route set and the convergence of the solution are also explained. Next, we present aggregate results concerning the route pattern on the road network, such as the number of OD pairs classified by the number of routes per OD pair. With this background, we then present more detailed analyses to consider how the number of OD pairs, and the total number of routes, in each class of routes per OD pair varies by OD generalized cost, OD flow and direction from the origin to the destination. For each analysis, selected graphs are presented to support our observations and conclusions. Interpretations of the findings and a brief conclusion complete the chapter.

\section{MODEL DESCRIPTION}

We consider a study area that is divided into a set of zones, $Z$, connected by transit services and by a road network. The road network consists of a set of nodes, $N$, and a set of directional links, $A$. In the Chicago regional model there are 1790 zones, 12,982 nodes and 39,018 links. A route is a sequence of nodes, $\left[v_{1}, \ldots, v_{k}\right]$, such that $\left[v_{i}, v_{i+1}\right] \in A$. The set of available routes from origin $p \in Z$ to destination $q \in Z$ is $R_{p q}$, and the set of all routes is $\mathbf{R}$.

The purpose of the model is to predict: (a) the mode-origin-destination (MOD) flow $d_{m p q}$, in persons per hour, for every origin $p \in Z$, destination $q \in Z$, and mode $m \in\{\mathrm{a}=$ auto, $\mathrm{t}=$ transit $\}$; (b) the distribution of auto OD flows to route flows $h_{r}$, for every route $r \in \mathbf{R}$. Auto OD flows are the sum of person-trips divided by a constant auto occupancy factor ( $a \circ f=1.2$ ) and the given OD truck flows $d_{p q}^{\text {truck }}$, in equivalent passenger cars per hour: $d_{p q}^{\text {auto }}=d_{a p q} / a o f+d_{p q}^{\text {truck }}$; hence $\sum_{r \in R_{p q}} h_{r}=d_{p q}^{a u t o}$. Total link flows are the result of route flow aggregation, $f_{a}=\sum_{r \in R: a \subseteq r} h_{r}$. A solution is feasible if it respects the constraints on total origin flows, $\sum_{m q} d_{m p q}=d_{\bullet p \bullet}$, and on 
total destination flows, $\sum_{m p} d_{m p q}=d_{\bullet \bullet q}$, where $d_{\bullet p \bullet}$ and $d_{\bullet \bullet q}$ are given inputs. In the model implemented for the Chicago Region, total MOD flows amount to 1.5 million $(1,513,211)$ persons per hour, while total truck flows amount to 0.4 million $(445,184)$ passenger-carequivalents per hour, representative of the morning peak period (6:30 - 8:30 am) in 1990 .

The remaining model inputs refer to transit and road levels of service. Transit data are invehicle travel time $c_{t p q}^{i v t t}$, out-of-vehicle travel time $c_{t p q}^{\text {ovtt }}$, and fare $c_{t p q}^{\text {fare }}$, for travelling from origin $p \in Z$ to destination $q \in Z$ by transit. These are fixed regardless of flows. Origindestination generalized cost by transit, $u_{t p q}$, is a weighted sum of the three components plus a constant bias; in the results reported here the transit bias is zero, and the weights are 0.25 , 0.90 , and 0.08 respectively.

Travel time by auto on link $a, t t_{a}$, is a function of total link flow, $t t_{a}\left(f_{a}\right)=t t_{a}^{0} \cdot\left(1+0.15 \cdot\left(f_{a} / k_{a}\right)^{4}\right)$, where $t t_{a}^{0}$ and $k_{a}$ are respectively the free-flow travel time and capacity of the link. Link generalized cost is $t_{a}=t t_{a}\left(f_{a}\right)+0.15 \cdot l_{a}$, where $l_{a}$ is the link length and the coefficient, 0.15 , reflects a combination of both the direct effect of distance on generalized cost and the indirect effect of fuel consumption. Fixed additional auto costs, $a c_{a p q}$, account for the parking fee and out-of-vehicle travel time at the origin and destination. The route generalized cost is $c_{r}=a c_{a p q}+\sum_{a \subseteq r} t_{a}$. The minimum OD generalized cost by auto is $u_{a p q}=\min \left\{c_{r}: r \in R_{p q}\right\}$. For every route $r \in R_{p q}$, define the excess cost as: $e c_{r}=c_{r}-u_{a p q}$. The user-equilibrium assumption is that the excess cost of every used route is zero. Approximate UE solutions are evaluated by the maximum excess cost over all used routes. MOD flows have the doubly-constrained Logit form, $d_{m p q}=A_{p} \cdot B_{q} \cdot \exp \left(-\mu \cdot u_{m p q}\right)$, with cost sensitivity $\mu=0.2$, and balancing factors, $A_{p}, B_{q}$, that ensure the constraints hold on total origin and destination flows. Approximate solutions of this model are evaluated by total misplaced MOD flow, $\sum_{m p q}\left|d_{m p q}-A_{p} \cdot B_{q} \cdot \exp \left(-\mu \cdot u_{m p q}\right)\right|$.

The combined model of user-equilibrium and mode-origin-destination choice with the specific structure described above can be formulated mathematically either as a fixed point problem, or as a convex optimization problem (Bar-Gera and Boyce, 2003). For the generalized link cost function stated above (separable, monotonically increasing), the equilibrium, or optimal, solution uniquely determines total link flows. Total link flows in turn uniquely determine link costs, route costs, and the set of minimum cost routes, referred to here as the set of UE routes. Of course, the route flows on this set of UE routes are not unique.

The model is solved by the OBA algorithm; route flow solutions are described by a set of restricting a-cyclic subnetworks $A_{p}$ for each origin, and origin-based approach proportions $\alpha_{p a} \in[0,1]$, such that $\alpha_{p a}=0$ for all $a \notin A_{p}$, and $\sum_{a \in A_{p}: a_{h}=v} \alpha_{p a}=1 \quad \forall p \in Z, v \in N, v \neq p$. The 
implicit set of routes from origin $p$ is the set of all routes within $A_{p}$, that is $R_{p q}\left\lfloor A_{p}\right\rfloor=\left\{r \in R_{p q}: a \subseteq r \Rightarrow a \in A_{p}\right\}$. The implicit route flows are given by $h_{r}=d_{a p q} \cdot \prod_{a \subseteq r} \alpha_{p a}$.

For the OBA algorithm, the number of UE routes per OD pair is unrelated to the number of iterations, unlike algorithms based on the method of Frank and Wolfe (1956).

The availability of route flows and approach proportions allows (a) adjustments of modeorigin-destination flows while retaining the current route proportions, (b) adjustments of restricting subnetworks to accommodate more routes, and (c) efficient adjustments to approach proportions that utilize second order derivatives of the objective function. The resulting algorithm offers precise convergence for large-scale networks. For the specific model of the Chicago Region presented here, the algorithm produced a solution with maximum excess cost of $1 \mathrm{E}-13$ equivalent auto in-vehicle minutes of travel time and total misplaced MOD flow of 1E-10 person-trips per hour.

By itself, a precisely converged solution does not necessarily guarantee a set of routes that is similar to the true, unique set of UE routes. As link flows and link costs converge towards their equilibrium values, so should excess costs. Therefore, excess costs of UE routes should decrease continuously towards zero, while the excess cost of any non-UE route should converge to a strictly positive value. The minimum equilibrium excess cost of all non-UE routes, considered as the rejection gap, is strictly positive as well. In principle, if a threshold below the equilibrium rejection gap is chosen, then at a certain finite level of convergence, the excess cost of all UE routes will be below the threshold, and the excess cost of all non-UE routes will be above the threshold.

We chose to include all routes with excess cost below a threshold of $2 \mathrm{E}-12$. The smallest excess cost of a rejected route is at least $30 \mathrm{E}-12$, which determines the estimated rejection gap. Therefore, there are no routes with $2 \mathrm{E}-12<$ excess cost $<30 \mathrm{E}-12$. There are several reasons to believe that the chosen set of routes is probably similar and perhaps identical to the true set of UE routes. One reason is the stability of the set of included routes in the final iterations of the origin-based assignment. Another reason is the order of magnitude difference between the chosen threshold and the estimated rejection gap. Additional reasons are discussed in Bar-Gera (2004), particularly the fact that the chosen set of routes maintains consistent consideration of alternative route segments, a fundamental property of sets of minimum cost routes in general and the set of UE routes in particular.

\section{Summary of the model of the Chicago Region}

To provide a general background for interpreting the findings for the route pattern, we present selected overall measures of the model solution for travel by auto, transit and truck in Table 1 . The table gives the total person flows by mode and vehicle flows by auto and truck between zone pairs (interzonal) and within zones (intrazonal), and the total generalized costs for the same categories. The mean values shown are the ratio of total costs to total flows. These generalized costs include the added costs of parking and out-of-vehicle time, whereas the 
costs shown in Figs. 5-8 below are based only on in-vehicle travel times and distances travelled. Therefore, the costs in the figures are somewhat lower than shown in Table 1.

Table 1. Summary Measures for the Chicago Region Model for the Morning Peak Period

\begin{tabular}{|c|c|c|c|c|c|c|c|}
\hline \multirow{2}{*}{$\begin{array}{c}\text { Hourly flows, } \\
\text { 6:30-8:30 am, } 1990\end{array}$} & \multicolumn{4}{|c|}{ Persons } & \multicolumn{3}{|c|}{ Vehicles } \\
\hline & auto & transit & total & $\% *$ & auto & truck & total \\
\hline \multicolumn{8}{|l|}{ Total flow (_/hour) } \\
\hline Interzonal & $1,116,689$ & 331,028 & $1,447,717$ & 23 & 930,574 & 418,507 & $1,349,081$ \\
\hline Intrazonal & 64,971 & 523 & 65,494 & 1 & 54,142 & 26,678 & 80,820 \\
\hline Regional & $1,181,660$ & 331,551 & $1,513,211$ & 22 & 984,717 & 445,184 & $1,429,901$ \\
\hline \multicolumn{8}{|l|}{ Total costs (hours) } \\
\hline Interzonal & 363,314 & 147,549 & 510,862 & 29 & 302,762 & 210,387 & 513,148 \\
\hline Intrazonal & 13,896 & 214 & 14,109 & 2 & 11,580 & 6,116 & 17,696 \\
\hline Regional & 377,209 & 147,762 & 524,972 & 28 & 314,341 & 216,503 & 530,844 \\
\hline \multicolumn{8}{|l|}{ Mean costs (min’s) } \\
\hline Interzonal & 19.5 & 26.7 & 21.2 & & 19.5 & 30.2 & 22.8 \\
\hline Intrazonal & 12.8 & 24.5 & 12.9 & & 12.8 & 13.8 & 13.1 \\
\hline Regional & 19.2 & 26.7 & 20.8 & & 19.2 & 29.2 & 22.3 \\
\hline
\end{tabular}

*percent transit $=100 \times($ transit/total $)$

Regional value $=$ Interzonal value + Intrazonal value

\section{DESCRIPTION OF THE ROUTE SET}

Using the procedure described above, we determined that the solution for the user-equilibrium route set has a total of $8,602,026$ routes connecting 3,202,310 zone pairs, or an average of 2.686 routes per OD pair; intrazonal travel is excluded from all calculations reported here. Of these zone pairs, $1,781,131$ or $56 \%$ are connected by only one route. The maximum number of routes between an OD pair in the solution is 1,920 from zone 628 to zone 32. The locations of these zones are marked on Fig. 1. The centroid of zone 628 is located 9.6 miles west of the Chicago Central Business District (CBD), somewhat west of Oak Park, Illinois. The centroid of zone 32 is located 11.2 miles north of the Chicago CBD in Evanston, Illinois. The airline interzonal distance is 12.8 miles. The direction of zone 32 from zone 628 is 32 degrees east of North. Effectively, no freeways or tollways serve this zone pair, as shown in Fig. 2.

The numbers of routes depend to a substantial extent on the level of congestion in the network. In two other solutions for the same model implementation with deterrence parameter values of 0.14 and 0.1 instead of 0.2 , the total numbers of routes are 11.5 million and 20.6 million, as compared with 8.6 million for this case; the maximum numbers of routes for a single zone pair are 21,360 and 5,088, respectively. With no congestion (fixed link costs), the UE solution is one route per OD pair, or 3.2 million routes, except in the unlikely event of exactly equal fixed route costs. 
Fig. 1 Zone System of the Chicago Region

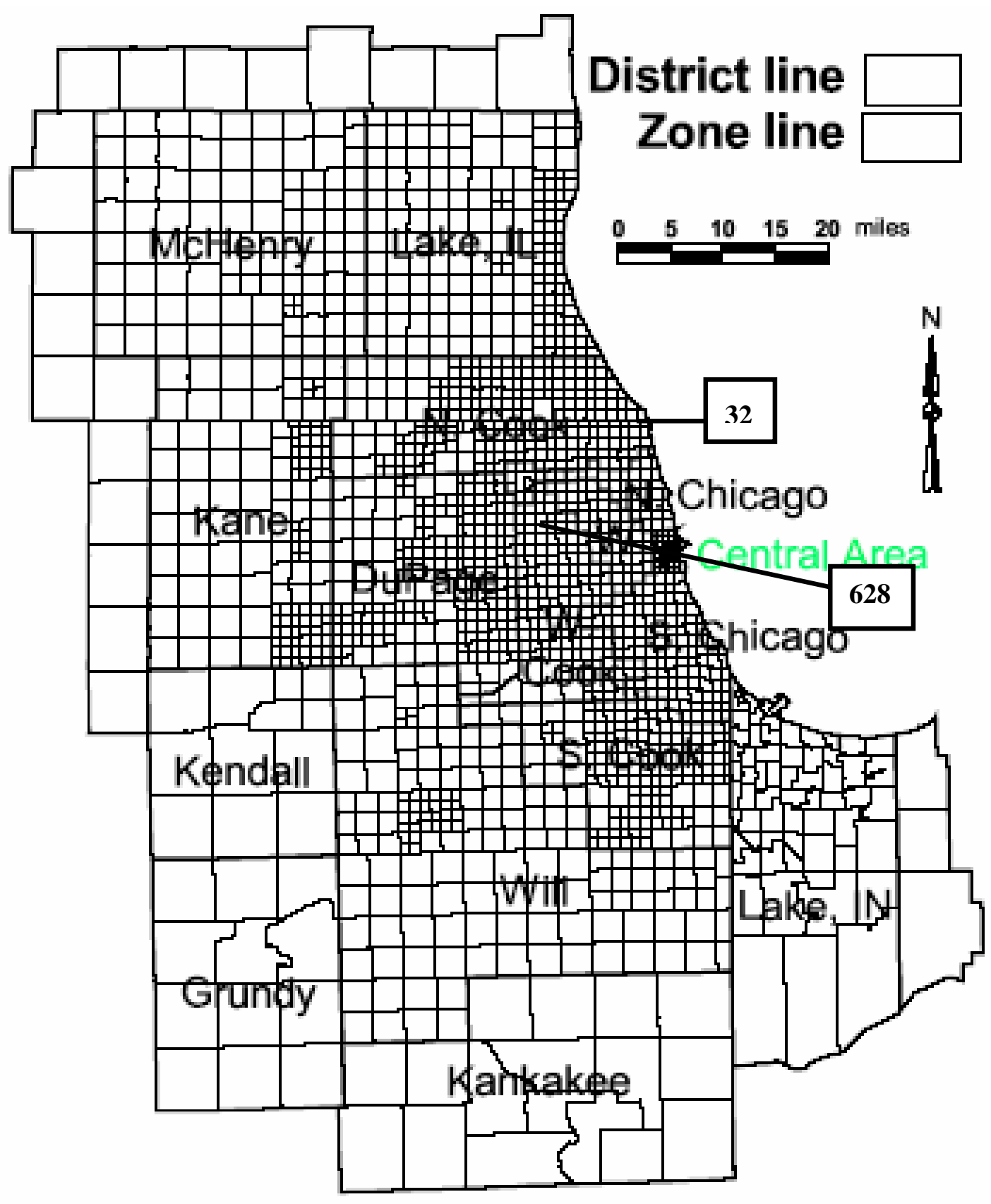


Fig. 2 Roadway Network of the Chicago Region

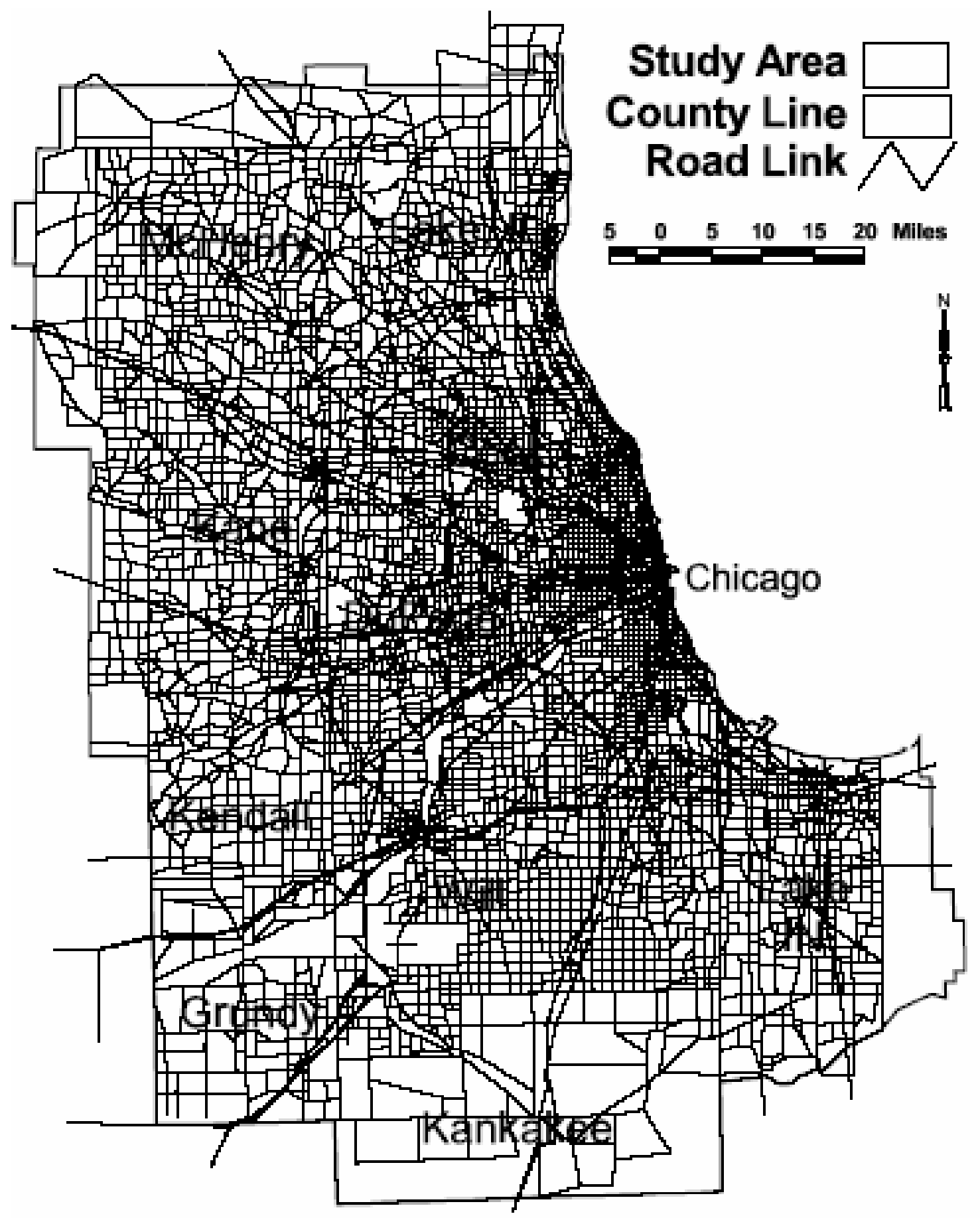




\section{Overall description}

Next, consider Fig. 3 showing the number of OD pairs with a specified number of routes, shown on the $\mathrm{x}$-axis ranging from 1 to 10,000. Two plots are shown on the figure: to the left is the number of OD pairs with each specified number of routes per OD pair; to the right is the cumulative proportion of OD pairs. For example, at the upper left, we can see there are nearly 1.8 million OD pairs with one route per OD pair, as noted above. The second point to the right and downwards indicates over 0.7 million OD pairs with two routes per OD pair. Continuing downwards to the right, we see decreasing numbers of OD pairs corresponding to increasing numbers of routes per OD pair, until we reach the rightmost point on the $\mathrm{x}$-axis with 1,920 routes per OD pair. By examining the figure closely, one may be surprised to see so many different numbers of routes per OD pair. The pattern is quite regular, with a wider range for the smaller number of OD pair counts.

Turning to the right axis, the cumulative proportion of OD pairs begins with $3.1 \mathrm{E}-7$ in the lower right corner for zone pair (628-32) and continues upwards and to the left ending with a cumulative proportion of 1.0 for one route per OD pair. By closely examining this aspect of Fig. 3, we can determine, for example, that about $90 \%$ of all OD pairs have five or fewer routes. This is not surprising, since $56 \%$ of all OD pairs have only one route. As noted above, this distribution depends to a substantial extent on the level of congestion in the network; the overall pattern shown in Fig. 3 represents a somewhat congested solution.

Another way of examining the same relationship is to consider the total number of routes with each number of routes per OD pair, as shown in Fig. 4. Clearly, the point in the upper left corner corresponding to OD pairs with only one route remains unchanged. However, the route count for OD pairs with two routes is doubled to 1.4 million, the number with three routes is tripled to 0.6 million and so forth. Those cases with only one OD pair, shown on the $\mathrm{x}$-axis of Fig. 3, now form a diagonal line rising to the right. The minimum total number of routes is now 47 , for the case of 47 routes per OD pair with only one occurrence.

As with Fig. 3, we also show the cumulative proportions of total routes in Fig. 4, beginning on the lower right with a value of 2.2E-4 for the case of 1,920 routes, rising to the left, and ending at 1.0 for one route per pair. From this figure we see that $90 \%$ of all routes connect OD pairs with 40 or fewer routes. Therefore, when examined from the viewpoint of total routes, OD pairs with as many as 40 routes become more important.

We suggest that these two figures convey interesting and important information about the route set on the Chicago road network. Naturally, these graphs raise further questions of how the number of OD pairs and the total number of routes vary with respect to the length, flow and direction of travel between OD pairs for various numbers of routes per OD pair. For example, are the origins and destinations connected by a single route primarily nearby? Or, are they OD pairs with very low flow? And what about the direction from origin to destination in relation to the grid-like arterial network of Chicago? We take up these interesting questions in the following subsections. 
Fig. 3. Number of OD Pairs by Number of Routes per OD Pair

Cumulative Proportion of OD Pairs

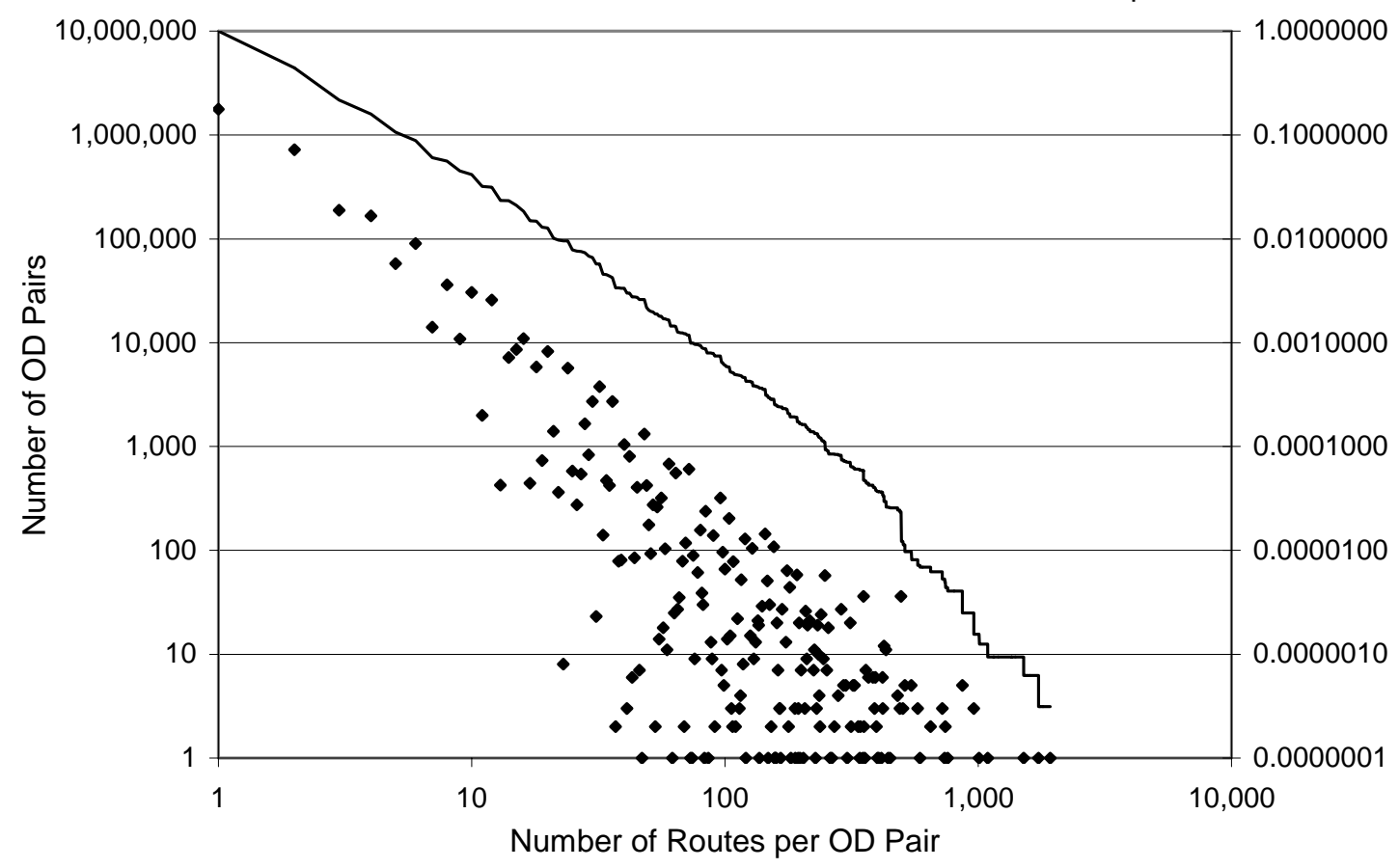

Fig. 4. Total Number of Routes by Number of Routes per OD Pair

Cumulative Proportion of Total Routes

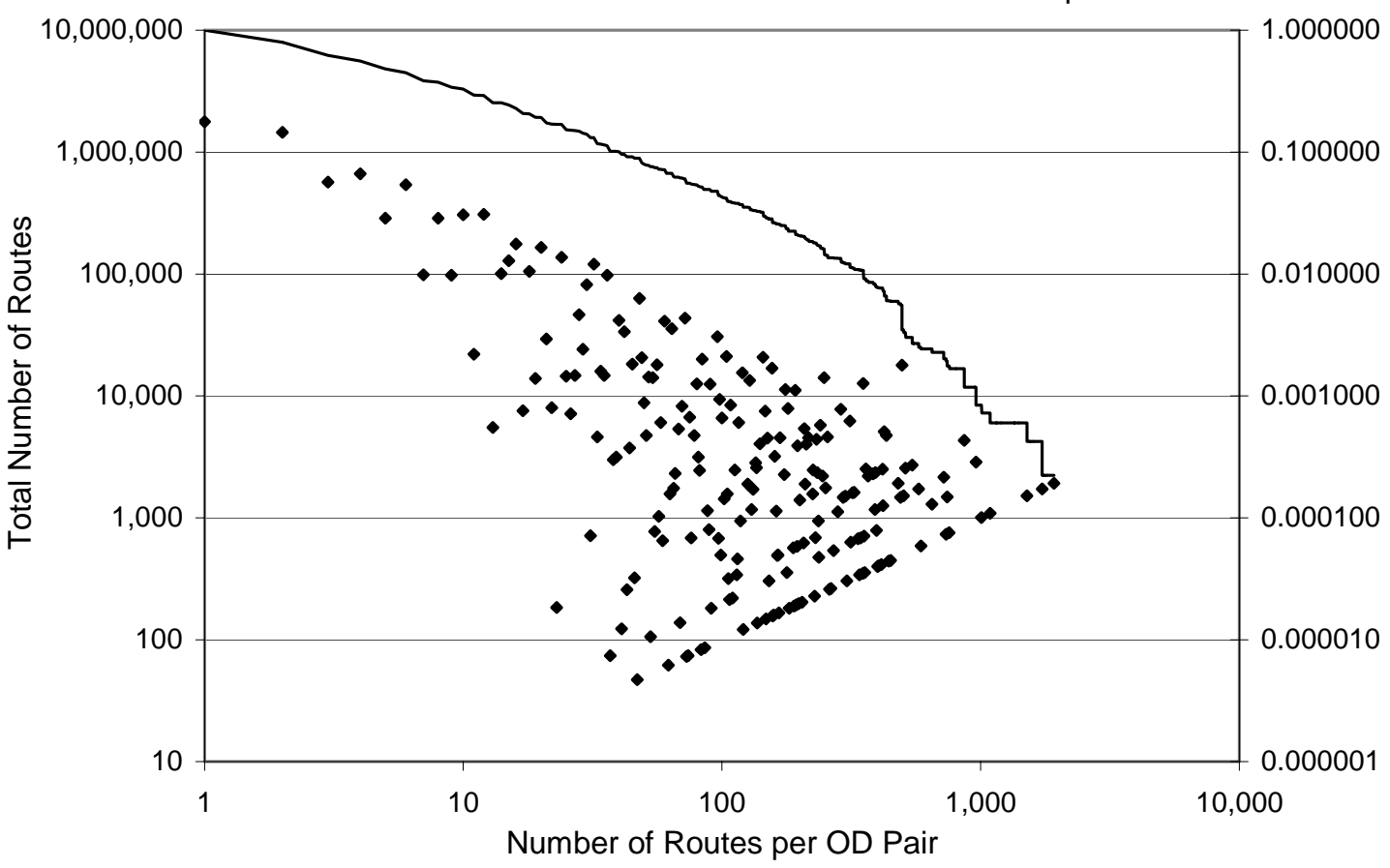




\section{Description of the route pattern with respect to cost}

To investigate the first question posed above, we present Figs. 5-8 relating the number of OD pairs and total number of routes to the generalized cost of travel between OD pairs. In each figure the $\mathrm{x}$-axis is the generalized cost throughout the network, which ranges from 0 to 200 auto in-vehicle minutes, the unit of cost defined by the model formulation. In Figs. 5 and 7, the $y$-axis is the number of OD pairs and the total number of routes, respectively. In Figs. 6 and 8, these numbers are converted to proportions within each unit increment of generalized cost. Note the labelling scheme in the figures: values for 1, 2 and 3-5 routes are shown in black with diamond, triangle and square symbols; this sequence is repeated in gray for 6-9, 10-99 and 100-1,999 routes. In each figure six classes of routes per OD pair are shown, which aggregate the route set based on more detailed analyses; see Table 2 for additional details.

Table 2. Number of OD Pairs and Total Routes by Classes of Routes per OD Pair

\begin{tabular}{|l|c|c|c|c|c|c|}
\hline $\begin{array}{l}\text { Routes per } \\
\text { OD pair }\end{array}$ & 1 & 2 & 3 to 5 & 6 to 9 & 10 to 99 & $\begin{array}{c}100 \text { to } \\
1,999\end{array}$ \\
\hline $\begin{array}{l}\text { Number of } \\
\text { OD pairs }\end{array}$ & $1,781,131$ & 724,829 & 412,279 & 150,810 & 131,306 & 1,955 \\
\hline $\begin{array}{l}\text { Total number } \\
\text { of routes }\end{array}$ & $1,781,131$ & $1,449,658$ & $1,517,572$ & $1,023,664$ & $2,460,034$ & 369,967 \\
\hline
\end{tabular}

Turning to Fig. 5, we observe that OD pairs in substantial numbers are present in the first five classes of routes over the full range of costs, although the numbers vary widely. The sixth class of routes $(100-1,999)$ has a smaller range (16 to 139 minutes) with smaller numbers of OD pairs (1 to 63 pairs, the largest occurring at a cost of 64 minutes). We find it remarkable that OD pairs with one route are found over the entire range of generalized costs from 1 to 184 minutes, with a few occurrences for even higher costs. The maximum count per unit cost value for 1-route OD pairs is 29,099 with a cost of 39 minutes. Similarly, substantial counts are found for 2 routes and the higher route classes. The general shape of these distributions is quite similar. The generalized cost for OD pair (628-32) is 44.4 auto in-vehicle minutes.

To provide information on how OD flows are related to OD costs, we show the proportion of total flow for each unit cost as a solid line extending from the lower right to the upper left. As expected, higher flows occur at lower costs. The scale for these flow proportions is given on the right y-axis. The highest flow proportion is 0.06 (6\%) of total flow at a cost of 8 minutes. Fig. 6 offers a different interpretation of these OD counts. The sum of OD pairs within each unit cost value is normalized to 1.0, in order to judge the relative size and importance of the route classes by cost. OD pairs with only one route dominate at lower costs, beginning with $98 \%$ of the total at a cost of 1 minute, but decline steadily to about $32 \%$ at 150 minutes where the pattern becomes rather chaotic. The next four classes (2; 3-5; 6-9; 10-99) increase very quickly at first, from being practically negligible at 1 minute to 19, 9, 3 and 2\% respectively at 20 minutes. Then they continue to increase gradually to 26, 26, 5 and $11 \%$ respectively at 150 minutes where all patterns become chaotic. Figs. 7 and 8 offer a comparable analysis in terms 
Fig. 5. Number of OD Pairs by Cost and Routes per OD Pair

Proportion of Total Flow

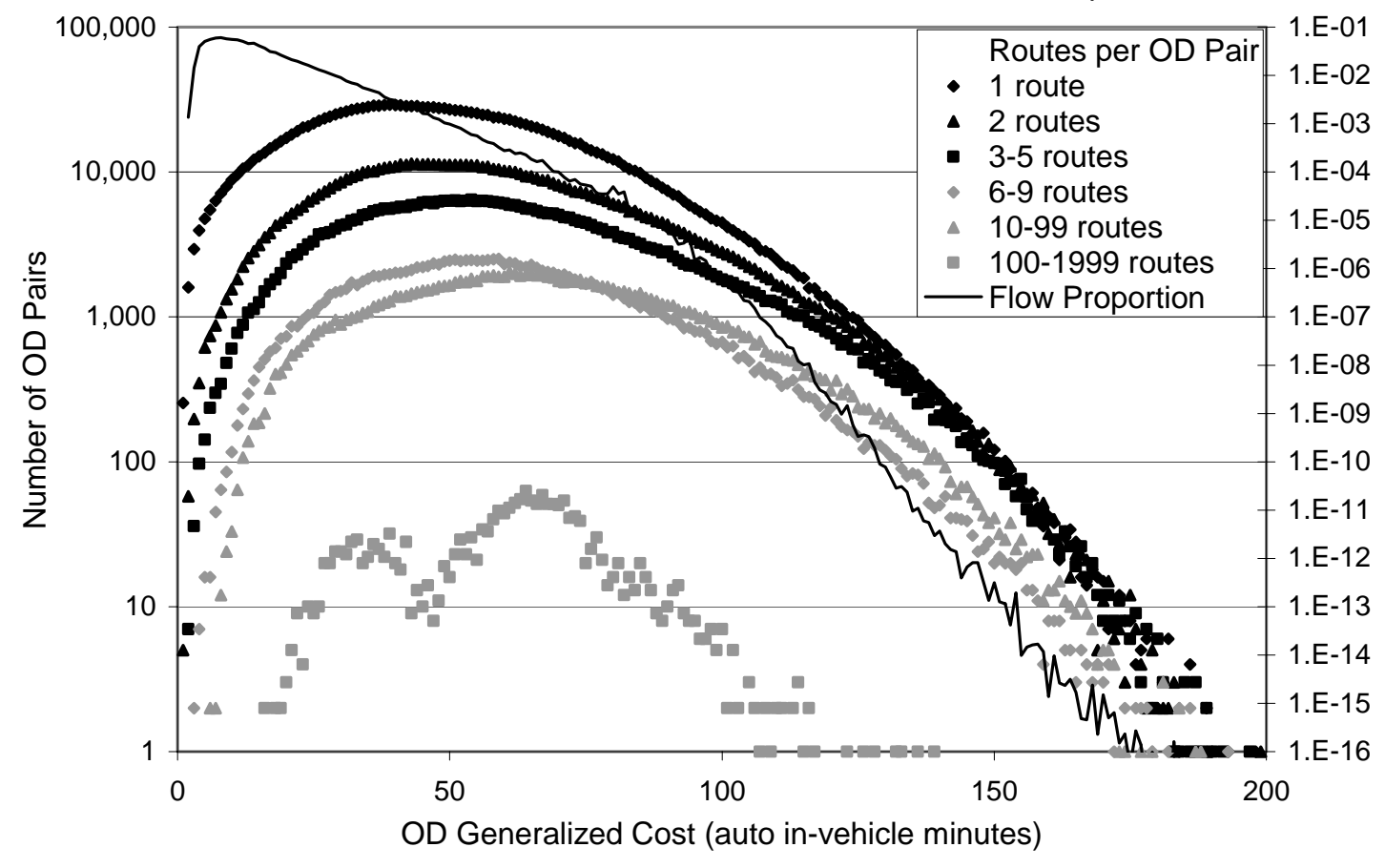

Fig. 6. Proportion of OD Pairs by Cost and Routes per OD Pair

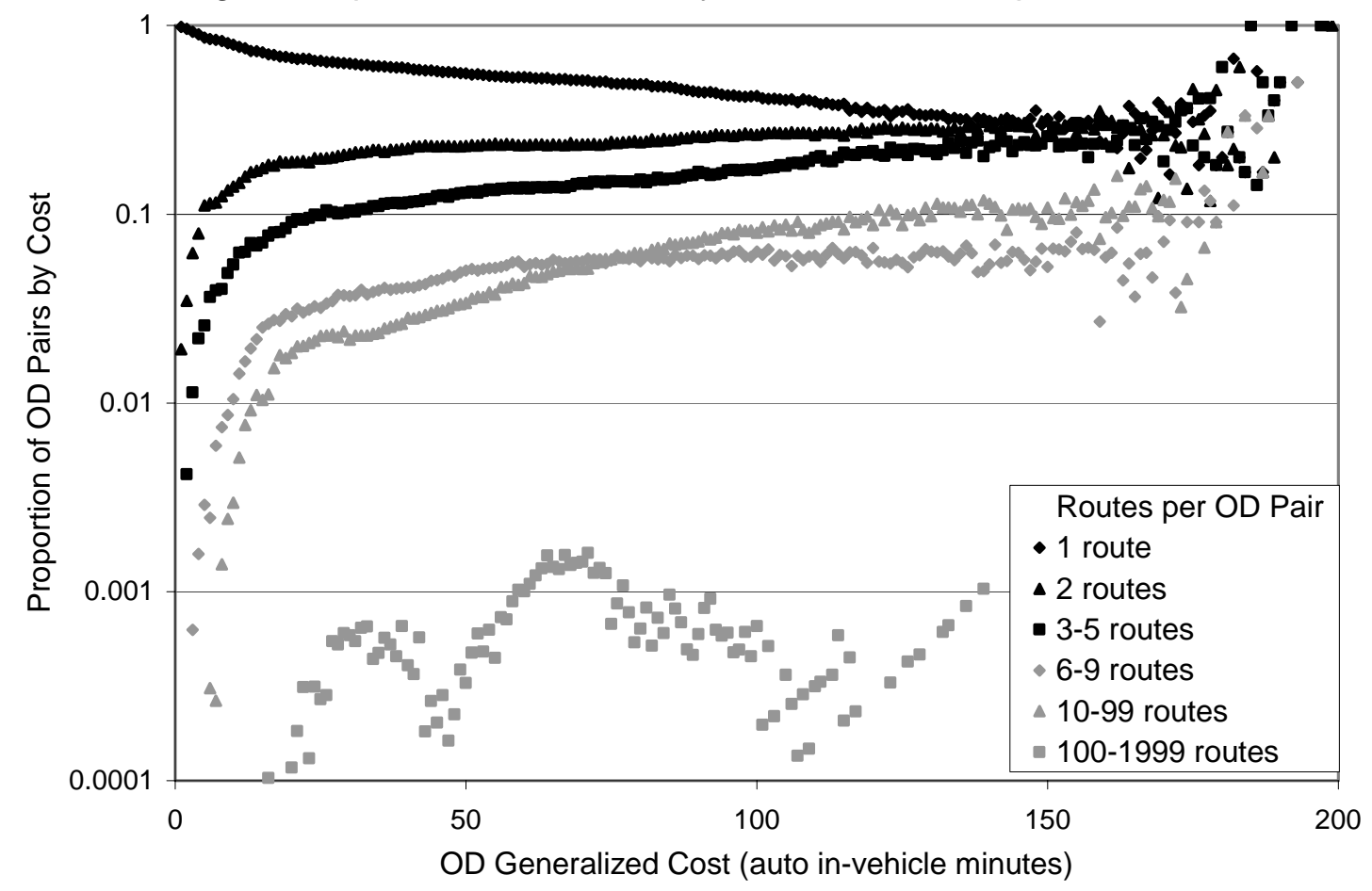


Fig. 7. Number of Routes by Cost and Routes per OD Pair

Proportion of Total Flow

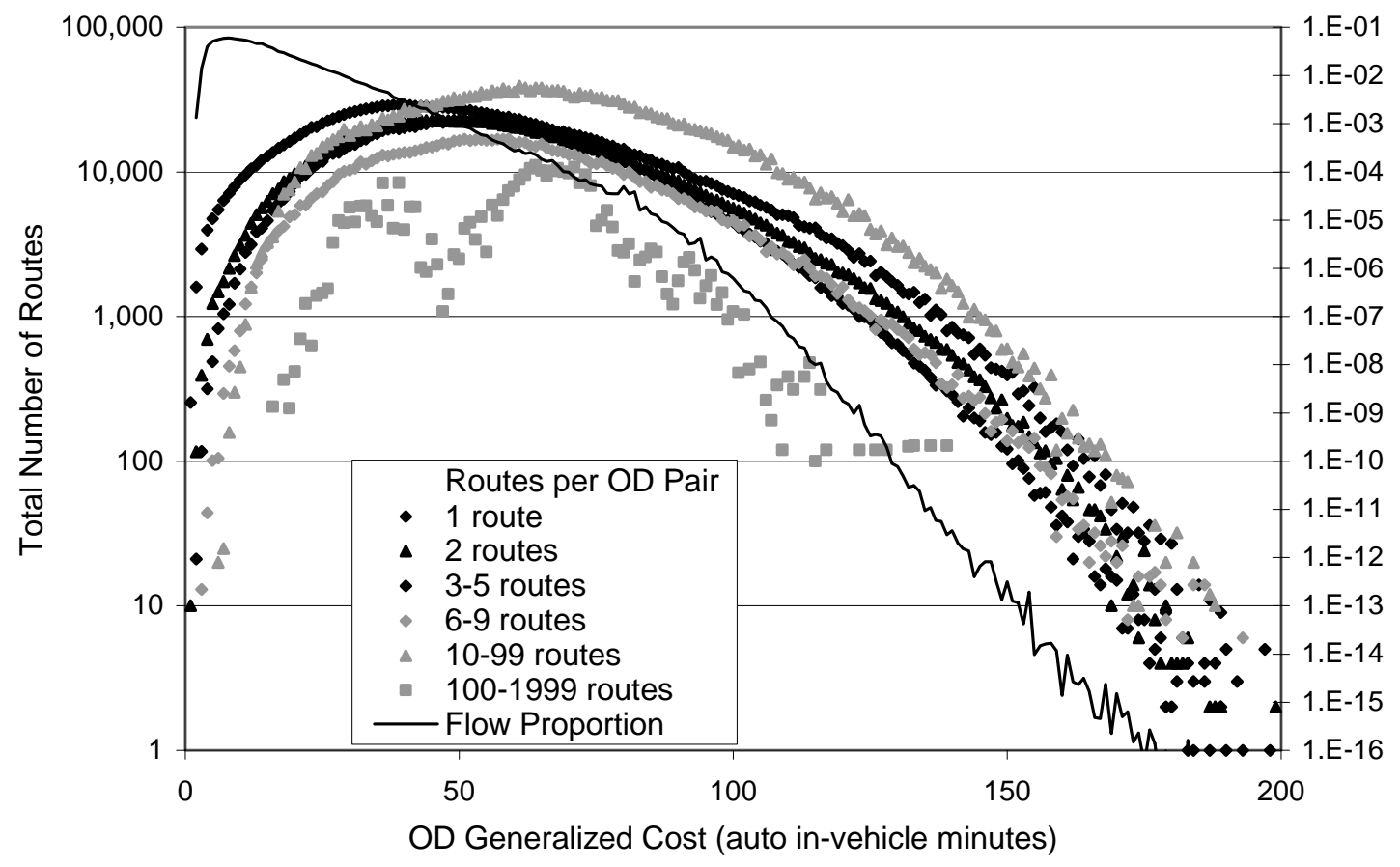

Fig. 8. Proportion of Total Routes by Cost and Routes

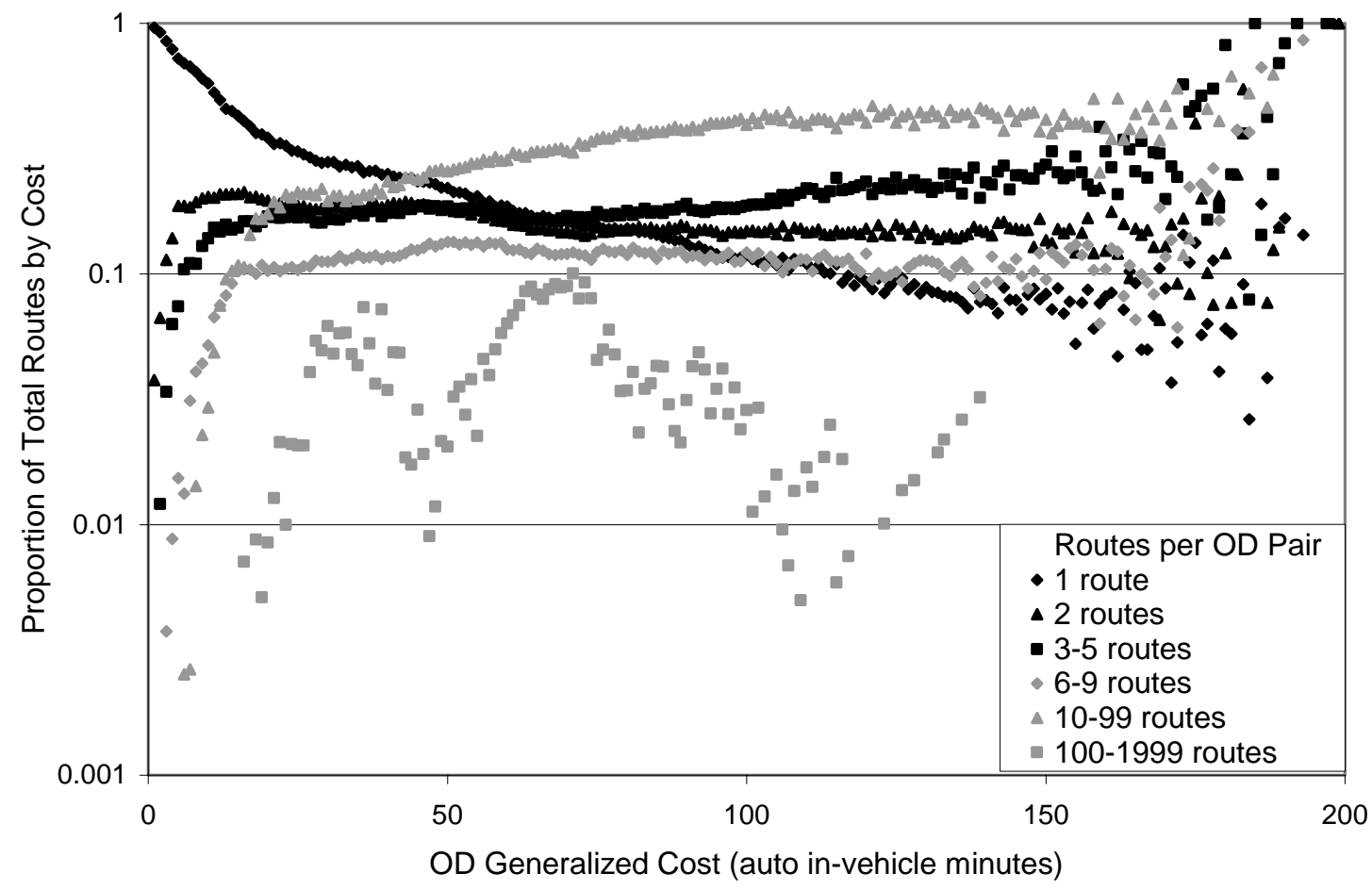


of the total number of routes. As shown in Fig. 7, the six route classes are similar in size and pattern. Total routes for OD pairs with 10 to 99 routes are the largest class.

\section{Description of the route pattern with respect to flow}

To investigate the second question posed above regarding the effect of OD flow on the number of routes, we present two figures relating the number of OD pairs, and total number of routes, to the flow of vehicles between OD pairs. In each figure the $\mathrm{x}$-axis is the OD flow per zone pair. The flows are shown in terms of orders of magnitude. For example, the value, -1 , represents flows from 0.1 (1E-1) up to but not including 1.0 (1E 0). The value, +3 , represents flows from $1,000(1 \mathrm{E}+3)$ up to 10,000 ; the maximum interzonal OD flow determined by the model is 2,186.1 vehicles per hour. There is one exception to this rule. At the left end of the $\mathrm{x}$-axis, the value, -14 , includes values from zero (0) up to $1 \mathrm{E}-13$. Hence, the range of the figure is from 0 up to 10,000. The definitions of the y-axis and classes of routes per OD pair are the same as Figs. 5-8.1

Since OD flows in the model are functions of cost, as defined in the Model Description, and small flows result from large costs, it is not surprising to find that Figs. 9 and 10 are essentially mirror images of Figs. 5 and 7. As with Figs. 5 and 7, the classes of routes extend across the full range of OD vehicle flows. However, the magnitude of the numbers of OD pairs and total routes are different because the level of aggregation on the $\mathrm{x}$-axis is 18 intervals of flow instead of 200 units of cost.

Also shown in Figs. 9 and 10 on the right y-axis, and by a dashed line, is the proportion of total flow, from the same data as in Figs. 5 and 7. Now the higher flows lie on the right, and decrease linearly on a log-log scale to the left, as flows decrease in magnitude. The number of OD pairs and total number of routes increases with flow, both as represented by order of magnitude and by total flow. In considering the magnitude of the increase, keep in mind the log scales. The reason for the larger number of OD pairs and total routes between zero and $1 \mathrm{E}-13$, as shown on the left axis, is that this flow interval includes zero OD flows. The model does determine the number of routes between OD pairs with zero flow; as can be seen, the number of routes is quite large in some cases. The substantial number of OD pairs with flows of less than one vehicle per hour results from the large number of zones $(1,790)$.

\section{Description of the route pattern with respect to direction}

As a final exploratory analysis, we consider the question of why many OD pairs have very few routes over the whole range of generalized cost and OD flow, while others have many routes, ranging in this case as high as 1,920 for one OD pair. As shown in Fig. 2, the arterial road network in the Chicago Region is relatively grid-like, especially in the city and inner suburbs where zones are small (one square mile or less) and the majority of zones are located. As an initial conjecture, we consider whether those zone pairs with only a few routes may be 
Fig. 9. Number of OD Pairs by Flow and Routes per OD Pair

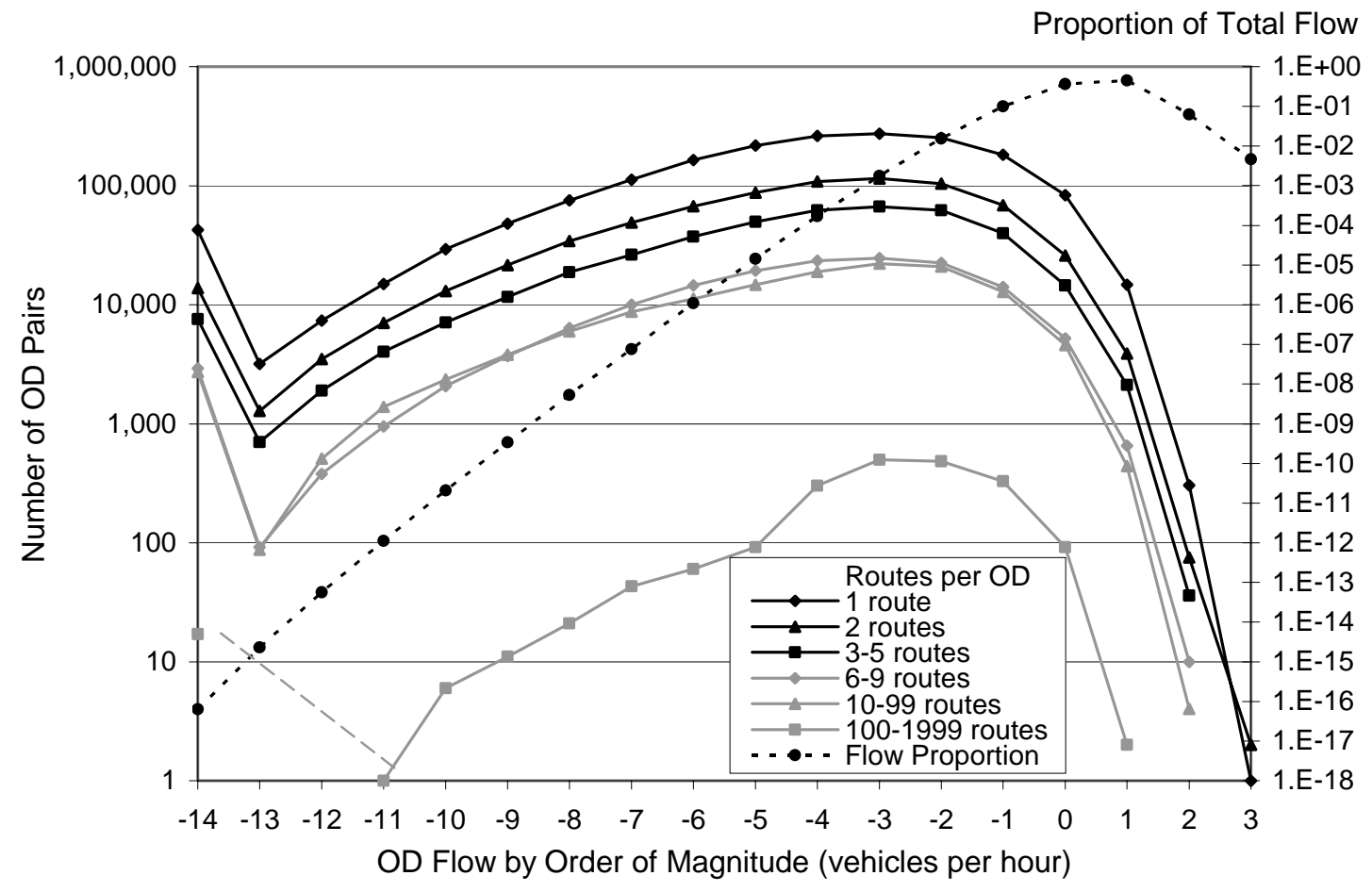

Fig. 10. Total Number of Routes by Flow and Routes per OD Pair

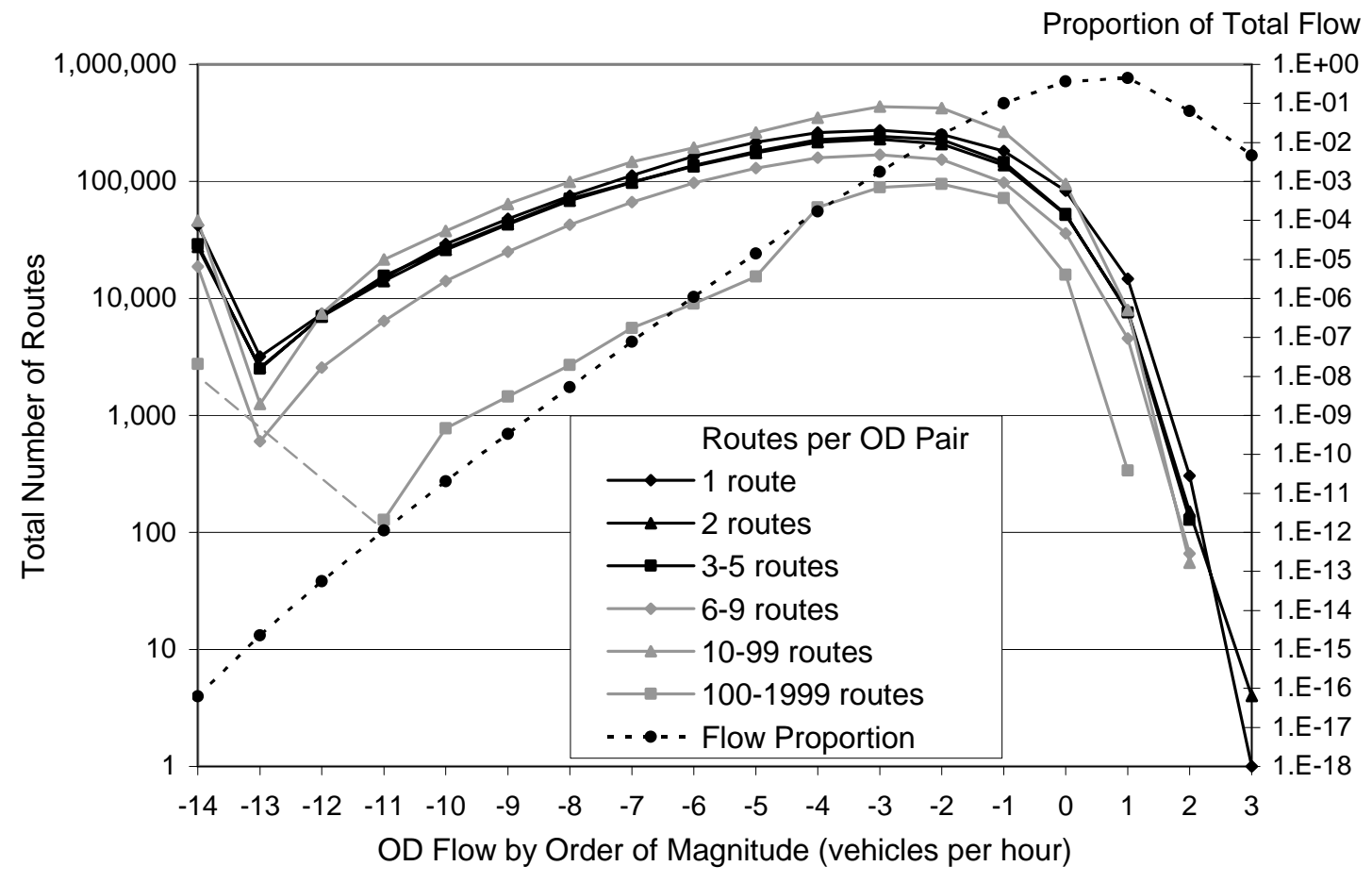


aligned with the grid (e.g. north to south or east to west), whereas zone pairs with larger numbers of routes may be aligned diagonally across the grid (e.g. southwest to northeast).

To examine this conjecture, we computed the direction of each destination from the origin, as measured from North. That is, if the destination lies directly north of the origin, its direction is 0 degrees. Sixteen directions were defined, each containing 22.5 degrees. Thus the direction representing south to north actually ranges from 348.75 degrees to 11.25 degrees. In Figs. 11 and 12, these 16 directions are shown on the x-axis by their midpoints, rounded to whole degrees. The 0 degrees direction is shown at the extreme left, and repeated at the extreme right as 360 degrees, to facilitate comparison with adjoining directions to the northwest. The principal compass points of North (0,360), East (90), South (180) and West (270) may be readily identified, together with NE (45), SE (135), SW (225) and NW (315); the values at the next level of detail are also represented, e.g., NNE (22.5). On the y-axis, we show the proportions of OD pairs and total number of routes by direction. These proportions are computed separately for each direction, as in Figs. 6 and 8. The definitions of the number of OD pairs and the total number of routes, as well as route classes, is identical to Figs. 3-10.

Examining the proportion of OD pairs by direction, we observe there does appear to be some support for our conjecture, especially for route classes 6-9 and 10-99. Class 100-1,999 supports the conjecture strongly for southbound travel (180 degrees) and westbound travel (270 degrees with no OD pairs), and somewhat for northbound (0 degrees) and eastbound travel (90 degrees). The pattern is emphasized more strongly in Fig. 12, showing the total number of routes. In this case, the values for 10-99 routes tend to support the conjecture, especially for travel towards the southeast and northwest directions. As noted earlier, OD pair $(628,32)$ with the largest number of routes $(1,920)$ has a direction of 32 degrees east of North, placing it in the second direction labelled 23 degrees; one of 103 OD pairs with 100 or more routes in the second direction, it represents about $7 \%$ of the routes in that class. Clearly, additional studies are required to reach more definitive conclusions, if warranted.

\section{INTERPRETATIONS}

As noted in the Introduction, this initial analysis of the model solution leaves many questions unanswered. Without repeating each of those questions here, we suggest that examining how these findings vary with road network congestion should have high priority.

A larger question concerns how one should interpret the OD, mode and route flow patterns and the route set underlying the user-equilibrium model, both with regard to the model formulation itself and with regard to reality. Given the large numbers of user-equilibrium routes in the solution, some readers may question the plausibility of the solution. Moreover, what is the meaning of route, mode and OD flows that are so very small?

From a computational viewpoint, these values are a result of the form of the doublyconstrained Logit function as well as the route choice model's user-equilibrium property. 
Fig. 11. Proportion of OD Pairs by Direction and Routes

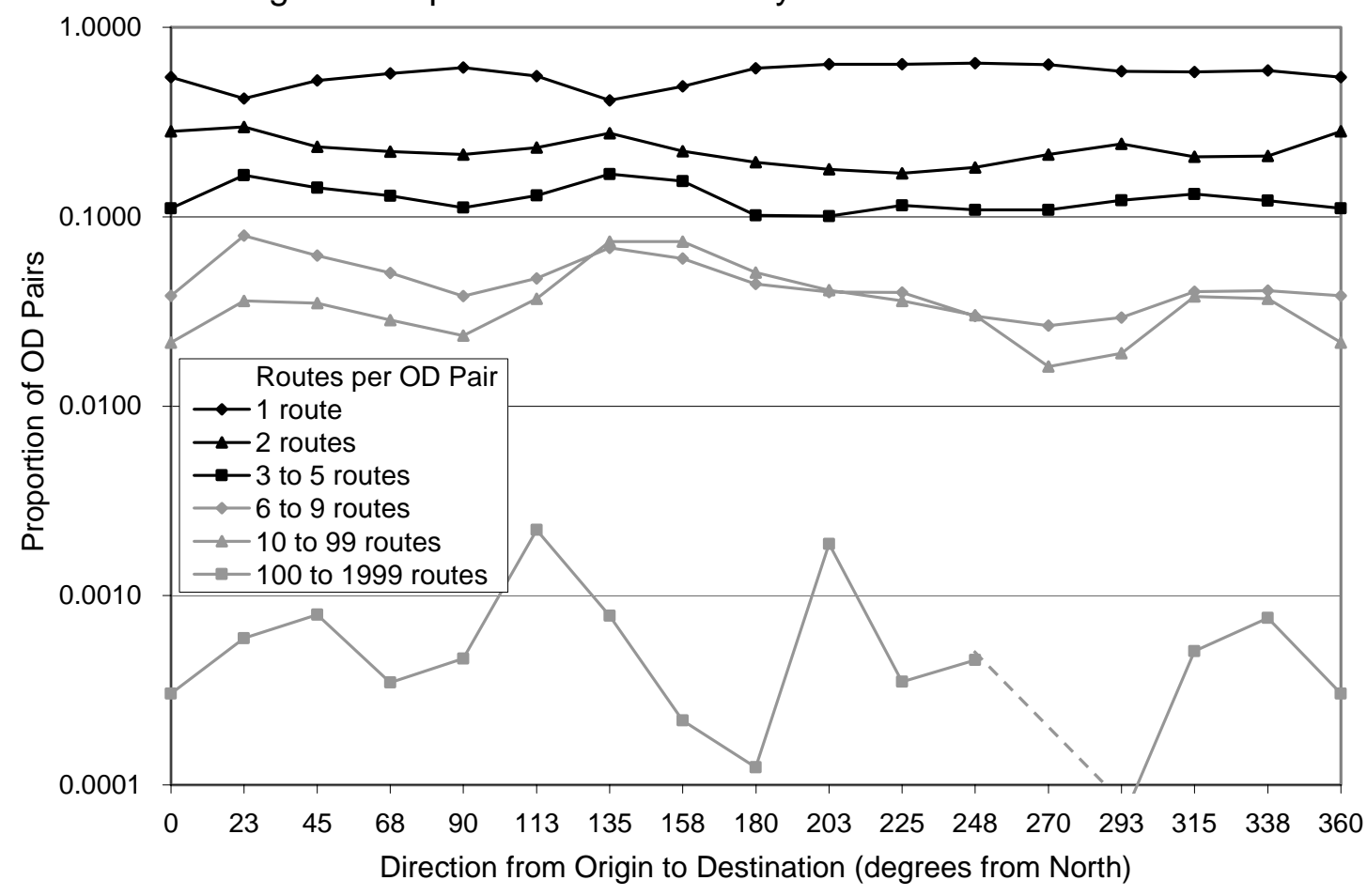

Fig. 12. Proportion of Total Routes by Direction and Routes

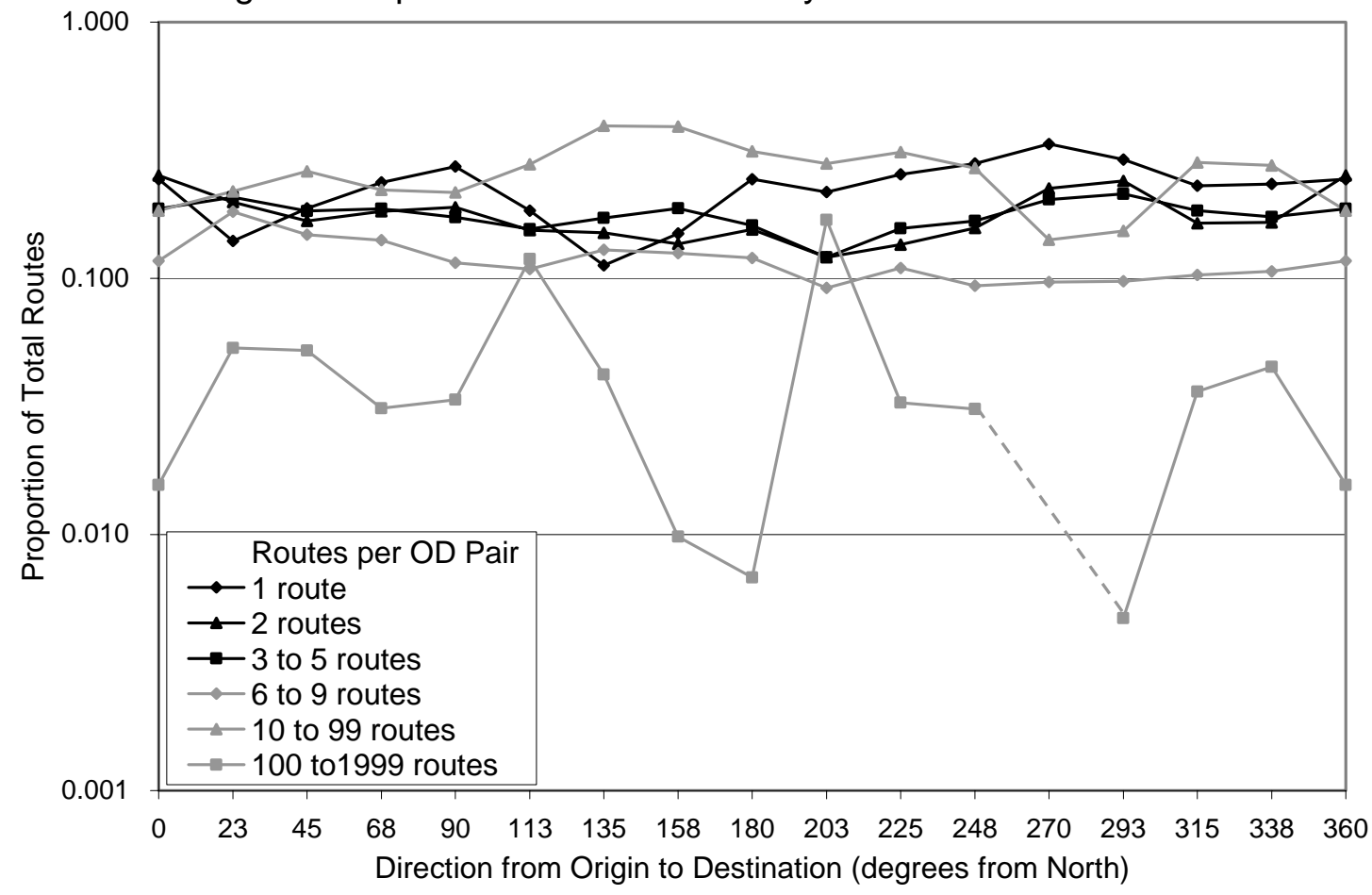


While the model could be truncated so as to exclude values less than some threshold, this restriction would change the properties of the model itself. Since our objective here is to understand the model's properties, such truncation would seem to be counterproductive. We suggest that the model solution should be viewed as an average or expected value, recognizing at the same time that flows on the order of $10^{-6}$ would require an enormously large number of replications to occur.

To achieve an impression of how the route flows might appear on a given day requires the application of a sampling procedure. That is, a random sample equal in size to the total number of trips per hour could be drawn from the MOD flow proportions and the route proportions. This sampling procedure would result in integer-valued MOD matrices as well as route flows, which could be summed to obtain link flows. Repeating this procedure perhaps 100 to 500 times would generate frequency distributions of link flows for each link, which could be examined to determine their means, range and variance and their correspondence to the deterministic link flows. This procedure would also answer the question of how many routes actually occur in a sampled realization for OD pairs with many routes. In examining these sampled realizations, we would need to keep in mind that no large-scale data set presently exists on route choice. In the absence of such data, we should be cautious in interpreting these results.

Beyond these immediate questions of assessing the model's validity is the relation of the model to travel forecasting practice. The model studied here is closely related to the sequential procedure, which remains the main paradigm of urban transportation planning practice. A difference from practitioner models is the assumption of a single class of travellers, but this restriction simply represents the current status of this research. Another shortcoming is the lack of a choice function for the time period of travel. A more general model could represent several time periods during the morning, and include a choice among them, based on relative levels of congestion as well as the cost of arriving too early or too late at the destination. Such a model would be more advanced than most practitioner models which typically apply survey-based factors to allocate 24-hour travel forecasts to shorter periods of time.

The model is also static, with all of the well-recognized limitations of that paradigm: continuous OD, mode and route flows from origin to destination; and non-decreasing link travel times in relation to link flows increasing without limit. Dynamic models of route, departure time, mode and OD choice have been the subject of ongoing research for nearly 30 years, and especially the last 15 years. Although dynamic models seek to represent the properties of road networks more realistically than their static counterparts, to our knowledge no dynamic model comes close to depicting the range of choices described by static, integrated models. For a recent state of the art review, see Peeta and Ziliaskopoulos (2001).

\section{CONCLUSIONS}

Our exploratory research has addressed for the first time the question of the characteristics of route sets in the solution to an integrated user-equilibrium model. Using graphical methods we have displayed the relationship of OD pairs and total routes in terms of generalized cost, 
OD flow and direction of travel, as well as the more aggregate relationships. We find the results surprising with respect to the broad range of values over which the classes of routes occur, and stimulating for gaining an improved understanding of the user-equilibrium model's properties.

\section{Acknowledgement}

The authors wish to thank Sanya Niumpradit for contribution to this chapter through his initial exploration of these questions in a term paper at the University of Illinois at Chicago in 2001, and Frederik Nöth for assistance with computations on which that term paper was based. Helpful questions and comments of four referees are gratefully acknowledged.

\section{REFERENCES}

Bar-Gera, H. (1999). Origin-Based Algorithms for Transportation Network Modeling. Technical Report 103, National Institute of Statistical Sciences, October, (Ph.D. thesis, Civil Engineering, University of Illinois at Chicago); downloadable file available at: http://www.niss.org/downloadabletechreports.html

Bar-Gera, H. (2002). Origin-based algorithm for the traffic assignment problem. Transportation Science, 36, 398-417.

Bar-Gera, H. (2004). Primal Method for Determining the Most Likely Route Flows in Large Road Networks. Submitted for publication in Transportation Science, September.

Bar-Gera, H. and D. Boyce (2003). Origin-based algorithms for combined travel forecasting models. Transportation Research, 37B, 405-422.

Beckmann, M., C. B. McGuire and C. B. Winsten (1956). Studies in the Economics of Transportation. Yale University Press, New Haven.

Bovy, P. H. L. and E. Stern (1990). Route Choice: Wayfinding in Transport Networks. Kluwer, Dordrecht.

Boyce, D. (2004). Forecasting Travel on Congested Urban Transportation Networks: Review and Prospects for Network Equilibrium Models. Presented at TRISTAN V, Guadeloupe.

Boyce, D. and H. Bar-Gera (2004). Multiclass combined models for urban travel forecasting. Network and Spatial Economics, 4, 115-124.

Brynooghe, M., A. Gilbert and M. Sakarovitch (1969). Une methode d'affectation du traffic. In: Proceedings of the 4th International Symposium on the Theory of Road Traffic Flow (W. Leutzbach and P. Baron, eds.), Beiträge zur Theorie des Verkehrsflusses Strassenbau und Strassenverkerhrstecknik, Heft 86, Herausgegeben von Bundesminister für Verkehr, pp. 198-204. Abteilung Strassenbau, Bonn, Germany.

Dafermos, S. C. (1968). Traffic Assignment and Resource Allocation in Transportation Networks. Ph.D. thesis, Operations Research, Johns Hopkins University, Baltimore.

Evans, S. P. (1973). Some Applications of Optimisation Theory in Transport Planning. Ph.D. thesis, Civil Engineering, University College London, London. 
Evans, S. P. (1976). Derivation and analysis of some models for combining trip distribution and assignment. Transportation Research, 10, 37-57.

Florian, M. and S. Nguyen (1976). An application and validation of equilibrium trip assignment methods. Transportation Science, 10, 374-389.

Florian, M. and D. Hearn (1999). Network equilibrium and pricing. In: Handbook of Transportation Science (R. W. Hall, ed.), pp. 361-393. Kluwer, Boston.

Frank, M. and P. Wolfe (1956). An algorithm for quadratic programming. Naval Research Logistics Quarterly, 3, 95-110.

Harris, B. (2002). Multiple paths through a network. Presented at the $42^{\text {nd }}$ Congress of the European Regional Science Association, Dortmund, Germany.

Jorgensen, N. O. (1963). Some Aspects of the Urban Traffic Assignment Problem. ITTE Graduate Report \#1963:9 (M.S. thesis, Civil Engineering), University of California, Berkeley.

Larsson, T. and M. Patriksson (1992). Simplicial decomposition with disaggregated representation for the traffic assignment problem. Transportation Science, 26, 4-17.

LeBlanc, L. J., E. K. Morlok and W. P. Pierskalla (1975). An efficient approach to solving the road network equilibrium traffic assignment problem. Transportation Research, 9, 309-318.

Lundgren, J. T. and M. Patriksson (1998). An algorithm for the combined distribution and assignment model. In: Transportation Networks: Recent Methodological Advances (M. G. H. Bell, ed.), pp. 239-253. Pergamon, Oxford.

Nagurney, A. (1999). Network Economics: A Variational Inequality Approach. Revised second edition. Kluwer, Boston.

Nguyen, S. (1974). An algorithm for the traffic assignment problem. Transportation Science, 8, 203-216.

Patriksson, M. (1994). The Traffic Assignment Problem - Models and Methods. VSP, Utrecht. Peeta, S. and A. Ziliaskopoulos (2001). Special Issue on Dynamic Traffic Assignment (Part I). Networks and Spatial Economics, 1, 231-390. 